\title{
Measurement of the Space Potential in Plasma
}

\author{
Kunio Hirao and Koh-ichiro Oyama \\ Institute of Space and Aeronautical Science, University of Tokyo
}

(Received October 20, 1969)

\begin{abstract}
The space potential in plasma is one of the fundamental quantity which should be determined in the diagnostics. The potential of a probe immersed in the plasma is determined on the basis of the space potential. Therefore, the determination of the space potential has been discussed long time since the method of D.C. probe was established. However, it has remained with much ambiguity for a precise discussion. In this paper, the problem of the determination of the space potential is studied from some different stand points. It is conclusively described that the space potential can be determined within the error of some millivolts.
\end{abstract}

\section{Introduction}

In the plasma diagnostics, determination of the space potential is one of the most important problems. For example, an electron density measurement with a Langmuir probe is made by obtaining the electron saturation current through the probe which is biased with the same potential as the space potential of the ambient plasma. The energy distribution of the plasma particles is also determined in the relation between the flux density of electrons and the voltage difference between the potential of a probe and the space potential of plasma.

In spite of this importance, the determination of plasma potential has still much ambiguity. Determination of the plasma potential by means of a Langmuir probe is considerably difficult due to a bending of the characteristic curve in the neighbourhood of the space potential as is well known to the plasma physicists. Kemp and Sellen (1966), Sato and Hatta (1962) and Dote (1966, 1968) described the emissive probe, the growing probe, the Twin probe and the RF probe methods, respectively, to determine the plasma potential. However these methods seem to be still not so accurate as to determine the plasma potential. Druyvesteyn (1930) proposed his ingeneous method to obtain the electron energy distribution. His method is the well-known second derivative method which determines the plasma potential from the second harmonic current in the probe circuit. He determined the space potential from the Langmuir curve. Later, Boyd and Twiddy (1958) suggested that the space potential corresponds to an inflexion point in the Langmuir characteristic curve and can be determined from the potential of probe when the second harmonic current through probe shows a minimum during a sweep of the bias voltage. Kilvington et al. (1967) suggested the same opinion as Boyed et al. Verobéva et al. (1962), on the contarary, considered that the potential of probe which manifests a maximum second harmonic current during the sweep of the 
bias voltage, might be the plasma potential.

Medicus (1968) presumed that the space potential might be between the dip point and the second maximum in the second harmonic experiment.

The problem in determining the space potential is so ambiguous as described here. Among these discussions, the dip point seems to be most likely the plasma potential. However the physical meaning of this minimum point was not yet thoroughly discussed. In this paper, this dip point is studied experimentally in connection with other experiments, i.e., the changes of the amplitude and phase of RF probe current, through a probe immersed in the plasma. These experimental results are also discussed theoretically.

\section{Theoretical Standpoint of Plasma Potential Measurement}

\subsection{Druyvesteyn analysis of second harmonic current of a probe in plasma}

Druyvesteyn (1930) showed that the electron energy distribution function $\phi(v)$ in the low-pressure gaseous plasma is shown as follows

$$
\phi(v)=\frac{2}{N A e}\left(\frac{2 m v}{e}\right)^{1 / 2} \frac{d^{2} i}{d v^{2}},
$$

where $i$ is a current through a probe in the plasma and is a function of probe voltage $v$, $i=f(v)$. $\quad N$ is an electron number density of plasma, $A$ is a surface area of probe, $e$ is an electronic charge, and $m$ is an electronic mass. $\phi(v) d v$ means the number of electrons in the unit volume of plasma which have the energy between $e v$ and $e(v+d v)$.

To obtain the function $\phi(v)$, one has to determine the second derivative of probe current in each probe voltage. Although the second derivative of the current-voltage characteristic curve can be obtained by graphical differentiation, more convenient and accurate methods will be proposed here.

When a small sinusoidal wave voltage is superposed on a probe immersed in the plasma, the current through the probe is generally expressed as follows

$$
\begin{aligned}
i=f(V+a \sin \omega t) & =f(V)+\frac{a^{4}}{4} f^{(2)}(V)+\frac{a^{4}}{64} f^{(4)}(V) \frac{a^{6}}{2304} f^{(6)}(V)+\cdots \cdots \cdots \\
& +\left[a f^{(1)}(V)+\frac{a^{3}}{8} f^{(3)}(V)+\frac{a^{5}}{192} f^{(5)}(V)+\cdots \cdots \cdots\right] \sin \omega t \\
& -\left[\frac{a^{2}}{4} f^{(2)}(V)+\frac{a^{4}}{48} f^{(4)}(V)+\frac{a^{6}}{1536} f^{(6)}(V)+\cdots \cdots \cdots\right] \cos 2 \omega t \\
& -\left[\frac{a^{3}}{24} f^{(3)}(V)+\frac{a^{5}}{384} f^{(5)}(V)+\cdots \cdots \cdots\right] \sin 3 \omega t+\cdots \cdots \cdots
\end{aligned}
$$

Here, $a$ is the amplitude of the superimposed sinusoidal voltage, $\omega$ is its angular frequency and $f^{(n)}$ is the $n$-th derivative of the current with respect to voltage.

Assume that the amplitude $a$ is very small, then the second harmonic current is expressed by

$$
i(2 \omega)=-\frac{a^{2}}{4} f^{(2)}(V) \cos 2 \omega t
$$

Therefore, the amplitude is expressed as $\left(a^{2} / 4\right) \cdot f^{(2)}(V)$, and becomes zero at the inflexion 
point of the current-voltage characteristic curve. When the amplitude of the second harmonic current is detected, it shows a sharp minimum at the inflexion point.

In the above-described treatment, the relation of current with voltage is assumed to be pure resistive and the capacitance of the ion sheath around the probe is not taken into account. Schematically, the ion sheath may be expressed as the parallel connection of $R_{S}$ and $C_{S}$ shown in Fig. 1. Here, $R_{S}$ and $C_{S}$ are the resistive and capacitive parts

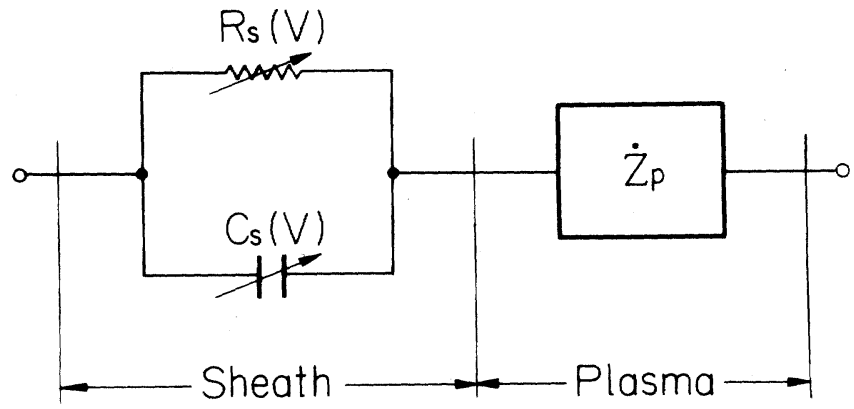

Fig. 1 The equivalent circuit of sheath-plasma impedance

of the sheath impedance respectively. The above described relationship (3) is, therefore, only valid for $R_{S}$, and is not the accurate description for the change of the physical state of the ion sheath. The effect of $C_{S}$ appears if the frequency of the superposed sinusoidal voltage is high as will be stated later. From this point of view, the ac signal of rather low frequency should be used in order to determine the electron energy distribution, which corresponds to the second derivative of the $i$-v curve, and higher frequency should be used for the determination of plasma potential as will be described later.

\subsection{Expression of the effect of ion sheath on the second harmonic analysis}

When the frequency becomes high, the effect of the capacitance of the ion sheath, $C_{S}$ on the total impedance of the probe becomes noticeable. The displacement current, $i_{d}$ due to this capacitance is as follows.

$$
i_{d}=C_{s} \frac{d V}{d t}
$$

As $C_{S}$ is a function of the probe bias, the general expression of $C_{S}$ is expressed as will be described later, when an ac signal of small amplitude is superposed on the $D C$ probe bias voltage.

Using again a series expansion,

$$
\begin{aligned}
C_{s}(V+a \sin \omega t) & =\left\{C_{s}(V)+\frac{a^{2}}{4} C_{s}{ }^{(2)}(V) \frac{a^{4}}{64} C_{s}{ }^{(4)}(V)+\cdots \cdots \cdots\right\} \\
& +\left\{a C_{s}^{(1)}(V)+\frac{a^{3}}{8} C_{s}{ }^{(3)}(V)+\cdots \cdots \cdots \cdot \sin \omega t\right. \\
& +\left\{\frac{a^{2}}{4} C_{s}^{(2)}(V)+\frac{a^{4}}{48} C_{s}^{(4)}(V)+\cdots \cdots\right\} \cos 2 \omega t+\cdots \cdots
\end{aligned}
$$

while the time derivative of the probe voltage is 


$$
\frac{d V}{d t}=\frac{d(V+a \sin \omega t)}{d t}=a \omega \cos \omega t
$$

Therefore, the displacement current is expressed as follows,

$$
\begin{aligned}
& i_{d}=\left\{C_{s}(V)+\frac{a^{2}}{4} C_{s}{ }^{(2)}(V)+\frac{a^{4}}{64} C_{s}{ }^{(4)}(V)+\cdots \cdots \cdots\right\} a \omega \cdot \cos \omega t \\
& +\left\{a C_{s}{ }^{(1)}(V)+\frac{a^{3}}{8} C_{s}{ }^{(3)}(V)+\cdots \cdots \cdots \cdots \cdots \cdots \cdots \cdots \cdots \cdots \cdots \cdots \cdot \sin \omega t \cdot \cos \omega t\right.
\end{aligned}
$$

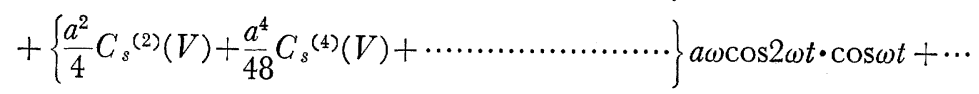

$$
\begin{aligned}
& =\left\{C_{s}(V)+\frac{a^{2}}{4} C_{s}{ }^{(2)}(V)+\cdots \cdots \cdot \cdots\right\} a \omega \cos \omega t+\frac{a^{2} \omega}{2}\left\{C_{s}{ }^{(1)}(V)+\frac{a^{4} \omega}{16} C_{s}{ }^{(3)}(V)+\cdots\right\} \\
& \sin 2 \omega t+\cdots \cdots \cdots \cdots
\end{aligned}
$$

The total sinusoidal current through the probe is the sum of the currents expressed by eq. (2) and (7). The current composed of the both components in the fundamental frequency by using each first term is expressed as follows,

$$
\begin{aligned}
& i(\omega) t=a f^{(1)}(V) \sin \omega t+C_{s}(V) a \omega \cos \omega t \\
& \quad=a\left[\left(f^{(1)}(V)\right)^{2}+\left(C_{s}(V) \omega\right)^{2}\right]^{1 / 2} \sin \left(\omega t+\theta_{1}\right)
\end{aligned}
$$

and

$$
\theta_{1}=\tan ^{-1} \frac{C_{s}(V) \omega}{f^{(1)}(V)}
$$

The current of the second harmonic frequency is expressed in the same way as follows,

$$
\begin{aligned}
i(2 \omega) & =-\frac{a^{2}}{4} f^{(2)}(V) \cos 2 \omega t+\frac{a^{2} \omega}{2} \sin 2 \omega t \cdot C_{s}{ }^{(1)}(V) \\
& =-\frac{a^{2}}{4}\left[f^{(2)}(V)^{2}+\left\{2 \omega C_{s}{ }^{(1)}(V)\right\}^{2}\right]^{1 / 2} \sin \left(2 \omega t+\theta_{2}\right) \ldots \ldots \ldots \ldots . .
\end{aligned}
$$

and

$$
\theta_{2}=\tan ^{-1} \frac{f^{(2)}(V)}{2 \omega C_{s}^{(1)}(V)}
$$

These equations, (8)-(11), show that the fundamental and second harmonic frequency components are controlled by $f^{(1)}$ and $f^{(2)}$ respectively in low frequencies, and $C_{S}$ and $C_{S}{ }^{(1)}$ in high frequencies. Physically, the mathematical differentiation of the $i$ - $v$ curve can be made when both ions and electrons move according to the externally applied field, as is considered in the $D C$ probe method, because $i$ - $v$ curve is determined in a steady state. Therefore, the frequency of the superposed sinusoidal wave should be lower than the ion plasma frequency in the ambient plasma, to measure the electron energy distribution. On the contrary, the frequency should be higher than the ion plasma frequency, but, of course, fairly lower than the electron plasma frequency to determine the plasma potential, because $C_{S}$ is maximum, and $C_{S}{ }^{(1)}(V)$ equals to zero, at the plasma potential as will be discussed later. 


\section{Experimental Study of the Second Harmonic Analysis}

\subsection{Test plasma and the experimental circuit}

The test plasma used for this experiment is the mercury plasma in a glass-made spherical tube of $30 \mathrm{~cm}$ diameter. The plasma are supplied from two back-diffusion type sources with hot cathodes which are mounted at two ends of a diameter of the spherical tube. This type of plasma source was developed by Takayama (1963). The probe used is a circular disc of $2 \mathrm{~cm}$ in diameter. The plasma tube is baked and evacuated up to a vacuum of $10^{-9}$ Torr before seal off the tube from the ultra high vacuum system. After that, a small mercury capsule which was put into the tube in advance, is broken by shock to fill the tube with mercury vapour. The vapour pressure of mercury, the number density of mercury molecule is controlled by temperature of mercury drop by means of the thermostat.

The present plasma tube has an electron density of $10^{6}-10^{7}$ els/cc and an electron temperature of $1000-2000^{\circ} \mathrm{K}$. The test plasma is quite stable so that the time change of plasma potential is about $2 \mathrm{mV}$ and the noise is less than a few millivolts.

The experimental circuit is shown in Fig. 2. The second harmonic current is picked up by means of a resistance $1 \mathrm{k} \Omega$, amplified selectively, detected and recorded in a $X-Y$ recorder with reference to the probe bias voltage.

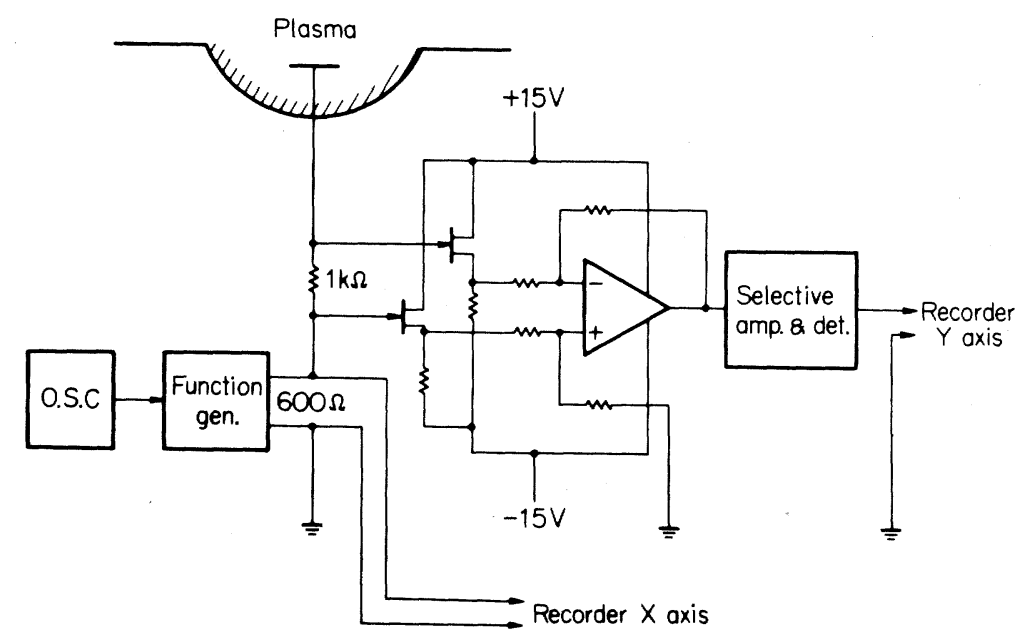

Fig. 2 Second harmonic component detecting circuit

\subsection{Experimental Result}

\subsubsection{Amplitude dependency of the probe bias at the dip point}

The second harmonic current is expressed as follows,

$$
i_{2 \omega}=-\left[\frac{a^{2}}{4} f^{(2)}(V)+\frac{a^{4}}{48} f^{(4)}(V)+\cdots \cdots \cdots \cdots \cdots \cdot \cos 2 \omega t\right.
$$


The ratio of the second term to first one is written as follows, assuming that the probe current has the exponential form like $i=i_{0} \cdot \exp \left(\frac{\mathrm{eV}}{k T e}\right)$, and the electron temperature is $1000^{\circ} \mathrm{K}$,

$$
\frac{a^{4} / 48 \cdot f^{(4)}(V)}{a^{2} / 4 \cdot f^{(2)}(V)}=\frac{a^{2}}{12}\left(\frac{e}{k T_{e}}\right)^{2} \doteqdot 1.1 a^{2}
$$

If the amplitude of the superposed ac signal is $0.1 \mathrm{~V}$, the effect of the derivative of the higher order on the second one is about $1 \%$. Figure 3 shows the deviation of the probe voltage at which the second harmonic current becomes minimum when the amplitude of the superposed ac signal is changed. When the amplitude of the ac signal is large, the potential at the dip point becomes higher than the correct potential. The correct potential can be obtained when the amplitude of the ac signal is less than few ten millivolts, this result being quite agreeable to that of eq. (13).

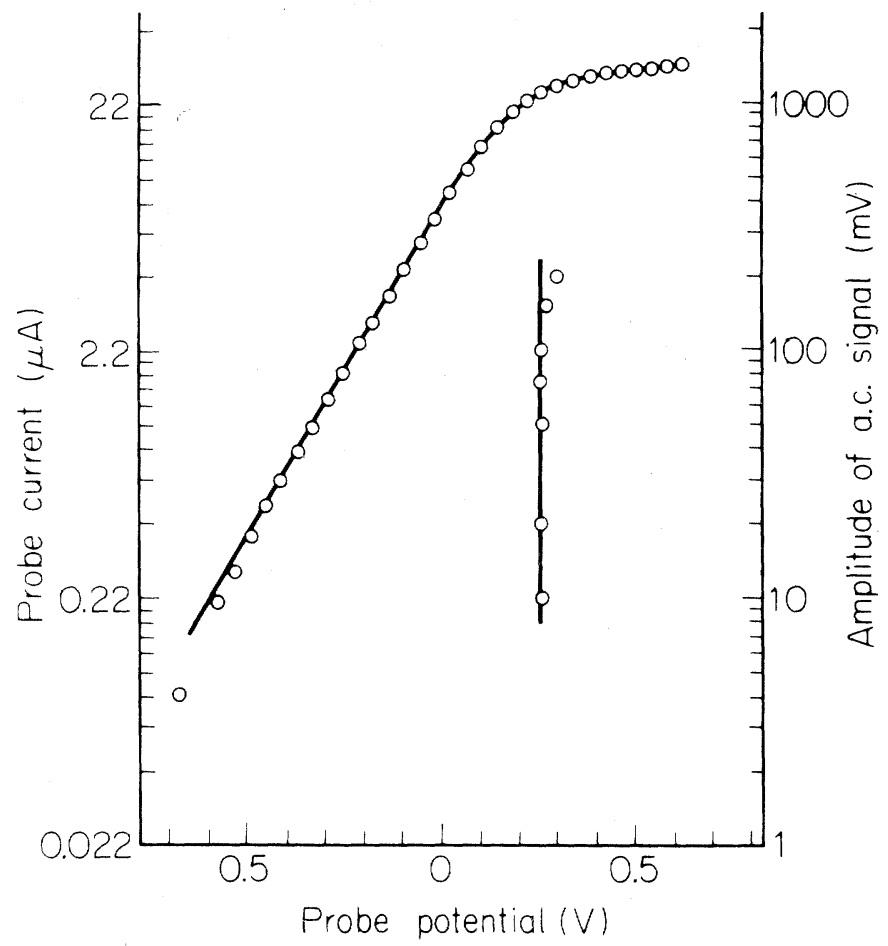

Fig. 3 Amplitude dependence of the minimum point of the second harmonic component. Ne: $6.7 \times 10^{6}$ electron $/ c c$, Te: $1900^{\circ} \mathrm{K}$ Langmuir $i$ - $v$ curve is simultaneously plotted.

\subsubsection{Frequency dependence of the probe bias at the dip point}

In this experiment, the amplitude of the input ac signal is fixed to be 60 millivolts to reduce the error on the second harmonic component to less than $1 \%$. The frequency of the ac signal is changed from $500 \mathrm{~Hz}$ to $50 \mathrm{kHz}$ to detect the change of characteristics of both the fundamental and second harmonic components of the ac current through the probe. Figure 4 indicates the characteristic curves showing the frequency dependence of 


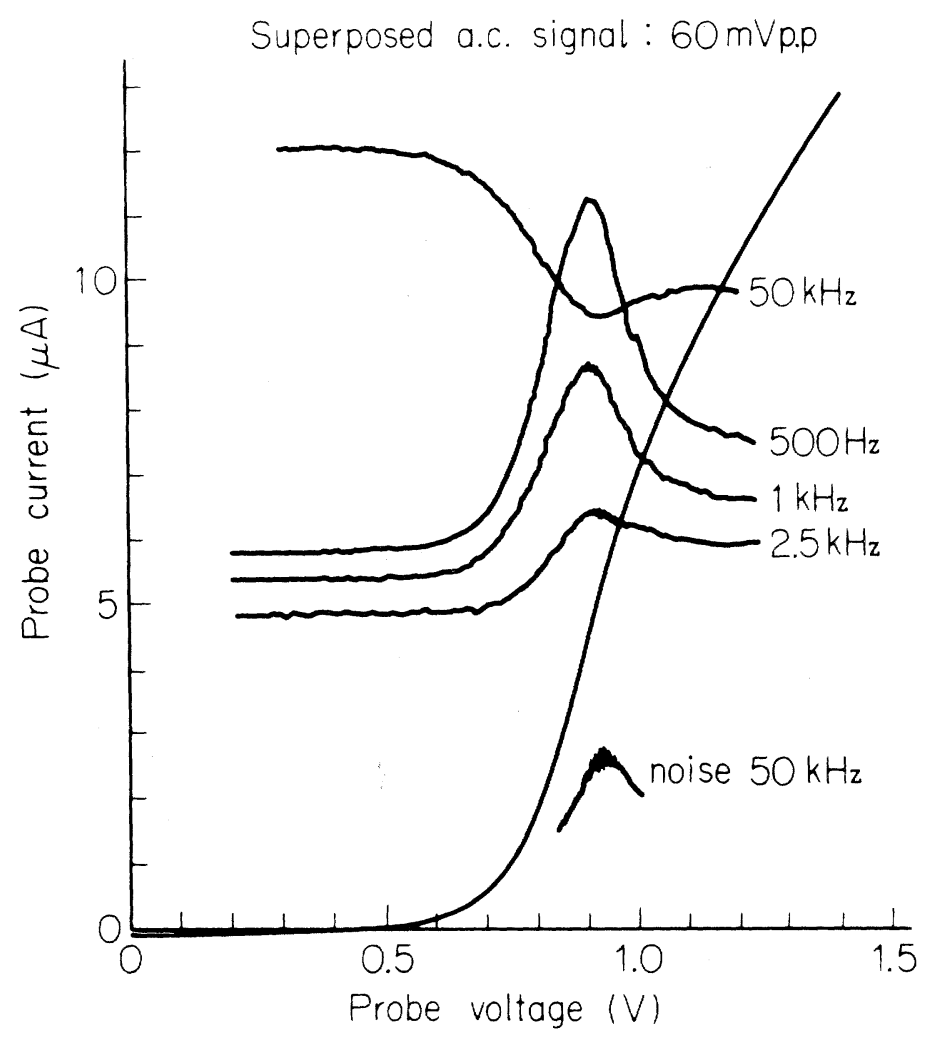

Fig. 4 Difference of fundamental component when the frequency of applied voltage is changed

the current of the fundamental component for both high and low frequency cases. Analogously Fig. 5 indicates the frequency dependence of the second harmonic component. From Fig. 5, one can observe that the second maximum is higher in the case of low frequency than in high frequency. This is understandable from the characteristics shown in Fig. 4. It can also be pointed out in Fig. 5 that the probe voltage at the dip point is slightly higher in the case of high frequency than in the case of low frequency. In this experiment, the probe voltage at the dip point is almost constant when the frequency of ac signal is higher than $15 \mathrm{kHz}$ as is shown in Fig. 7. This means that the effect of the ion sheath around the probe becomes dominant at higher frequency. To make these relations clear, Fig. 6 indicates the relation between the probe bias voltage at the dip point versus the amplitude of the superposed ac signal with frequency as a parameter. This relation shows that the probe voltage at the dip point changes more sensitively with amplitude of the superposed alternating voltage at higher frequency. Relations between the probe bias voltage at the dip point and the frequency applied to the probe for various plasma densities, which are controlled by changing the discharge current in the plasma sources, are shown in Fig. 7. The curves for different discharge currents are normalized in the low frequency range. From this figure, it is noticed that 
the probe bias voltage at the dip point is almost constant both in the low and high frequency range. The frequency where the transition from the low to the high frequency range occurs becomes higher when the plasma density becomes larger. This transition seems to take place around the ion plasma frequency of the experimental plasma.

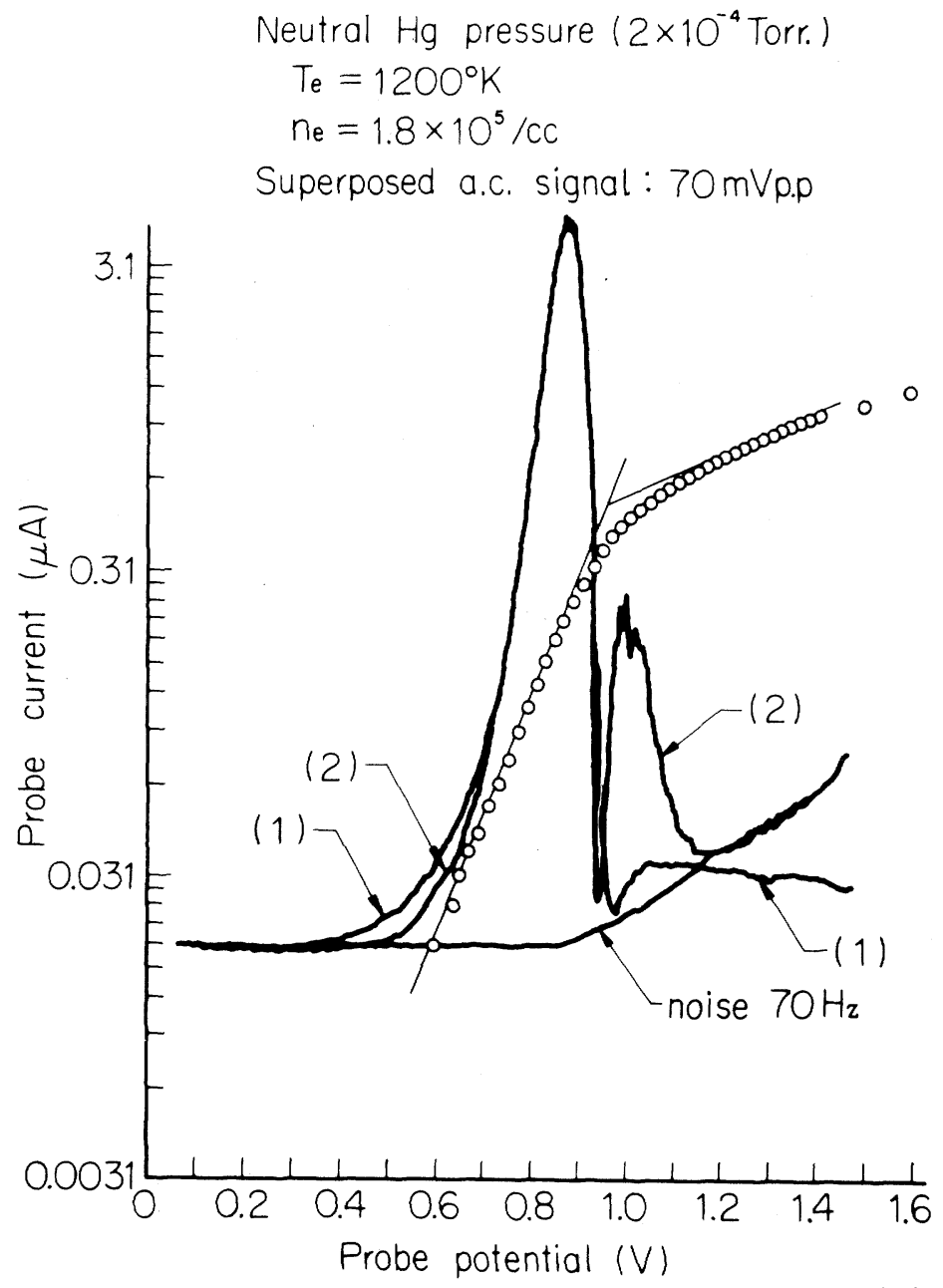

Fig. 5 Frequency dependence of the second harmonic current (1) 25 $\mathrm{kHz}$, (2) $70 \mathrm{~Hz}$ 


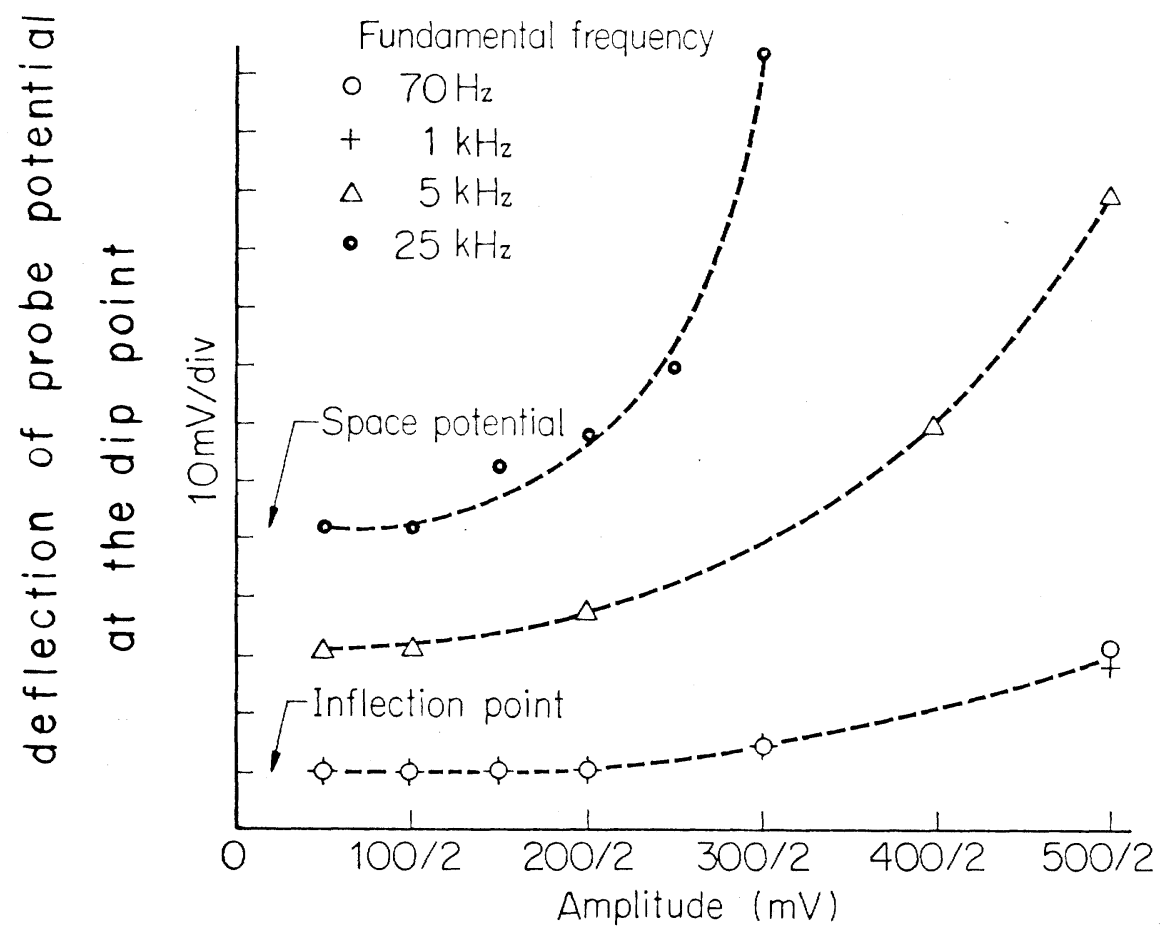

Fig. 6 Amplitude dependence of the dip point when the frequency of the superposed voltage is changed. Ne: $7.8 \times 10^{5}$ electron $/ c c, T e: 900^{\circ} \mathrm{K}$

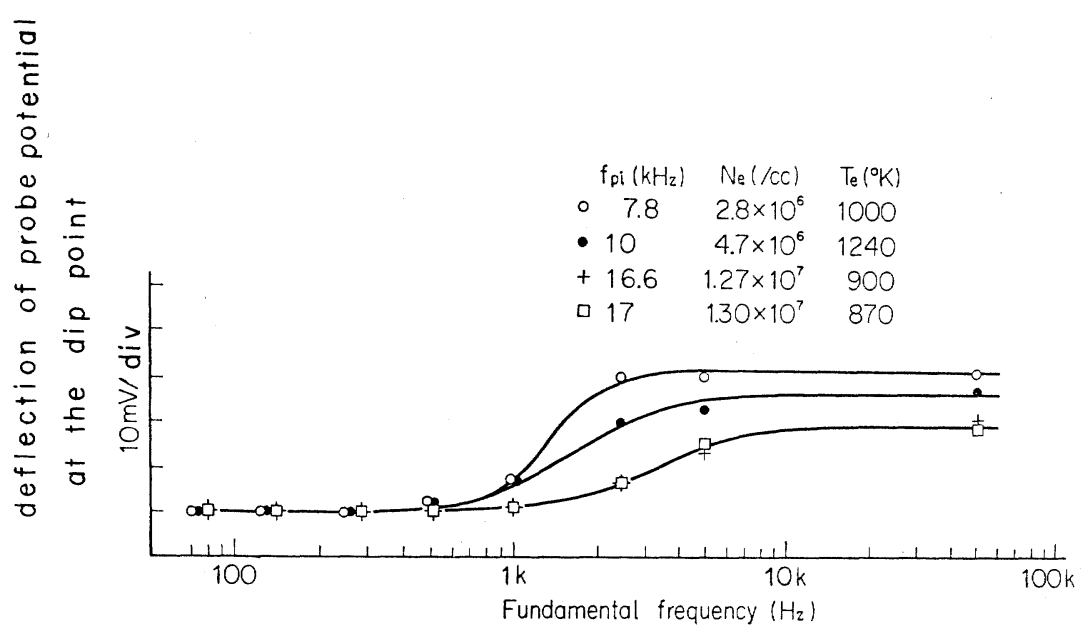

Fig. 7 Frequency dependence of the dip point when the electron density is taken as parameter 


\section{Experimental Study of the Phase Difference between Applied Voltage and Output Gurrent on a Probe}

The theoretical standpoint of the experiment in this section is eq. (9) in the previous paragraph 2.2. In the ideal case, the phase difference at the space potential might be about $0^{\circ}$ or minimum, because the denominator is expected to become very large compared with the increase of the numerator in eq. (9) at the space potential. The experimental circuit shown in Fig. 8 is used, in which the input circuit is a FET transistorized

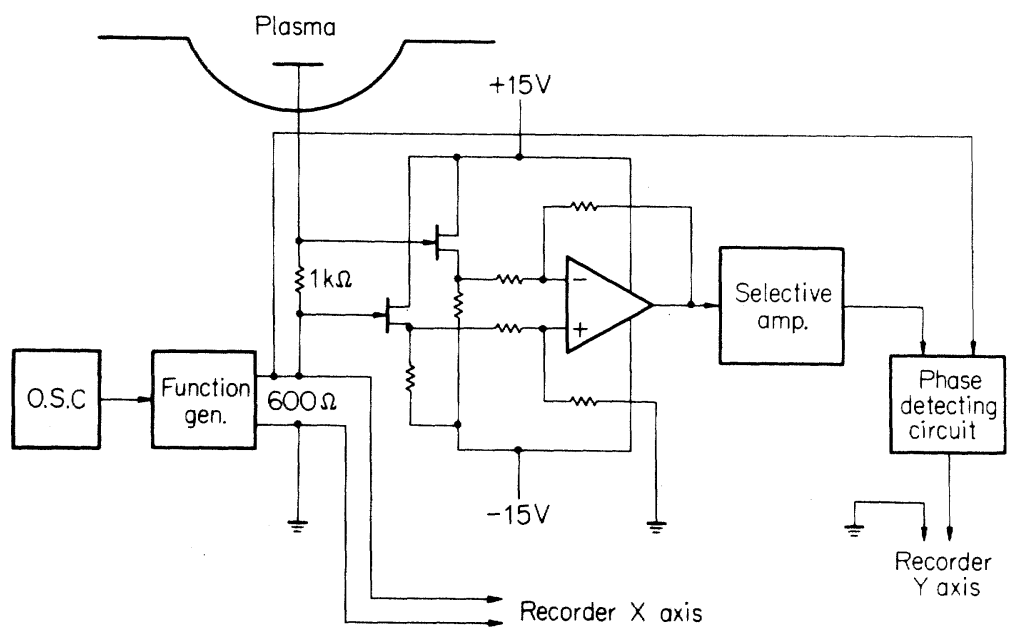

Fig. 8 Experimental circuit for phase defference detecting

differential amplifier to avoid the disturbance due to the measuring circuit. The test plasma used is the same as that in the previous experiment, and the frequency range used is from $500 \mathrm{~Hz}$ to $20 \mathrm{kHz}$. The first result is a relation between the phase difference and the frequency of the ac voltage of a constant amplitude applied to the probe. Figure 9 shows the result that the probe potential at which the phase difference becomes minimum, increases as the frequency of the ac-signal increases and approaches to a definite potential as in the case of Fig. 7. Figure 10 shows the relation between the phase difference and the applied frequency at the probe potential of $0.8 \mathrm{~V}$ and $2.5 \mathrm{~V}$, respectively. It shows a linear relationship between these two quantities, expressing that the theoretical relation of eq. (9) is appropriate to explain the experimental result. The probe capacitance, $C_{S}$, in the eq. (9) is calculated by means of the experimental results. The calculated capacitance is larger than that expected from the theoretical treatment of sheath capacitance. The discrepancy might be due to a contamination of the surface of the probe. The second results is the relation between the phase difference and the amplitude of the applied sinusoidal voltage, which is shown in Fig. 11 with the parameter of the amplitude. In this case, the dip point does not change so clearly as in the case of second harmonic analysis. 


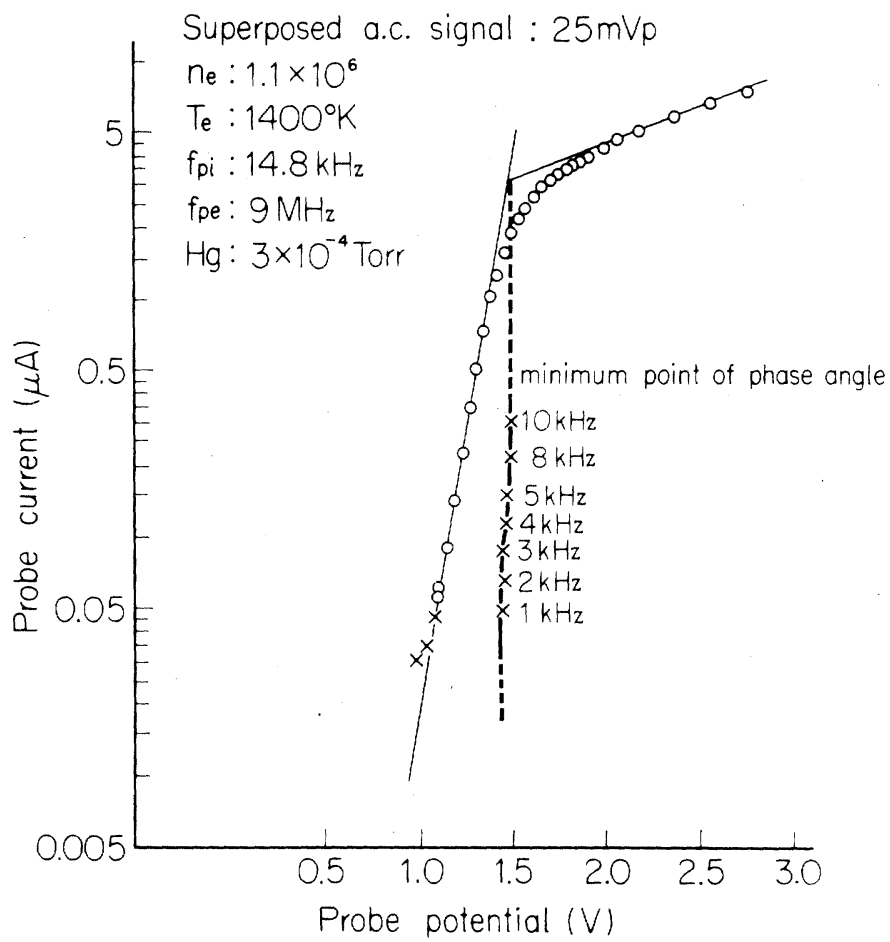

Fig. 9 Frequency dependence of the point where phase difference shows minimum

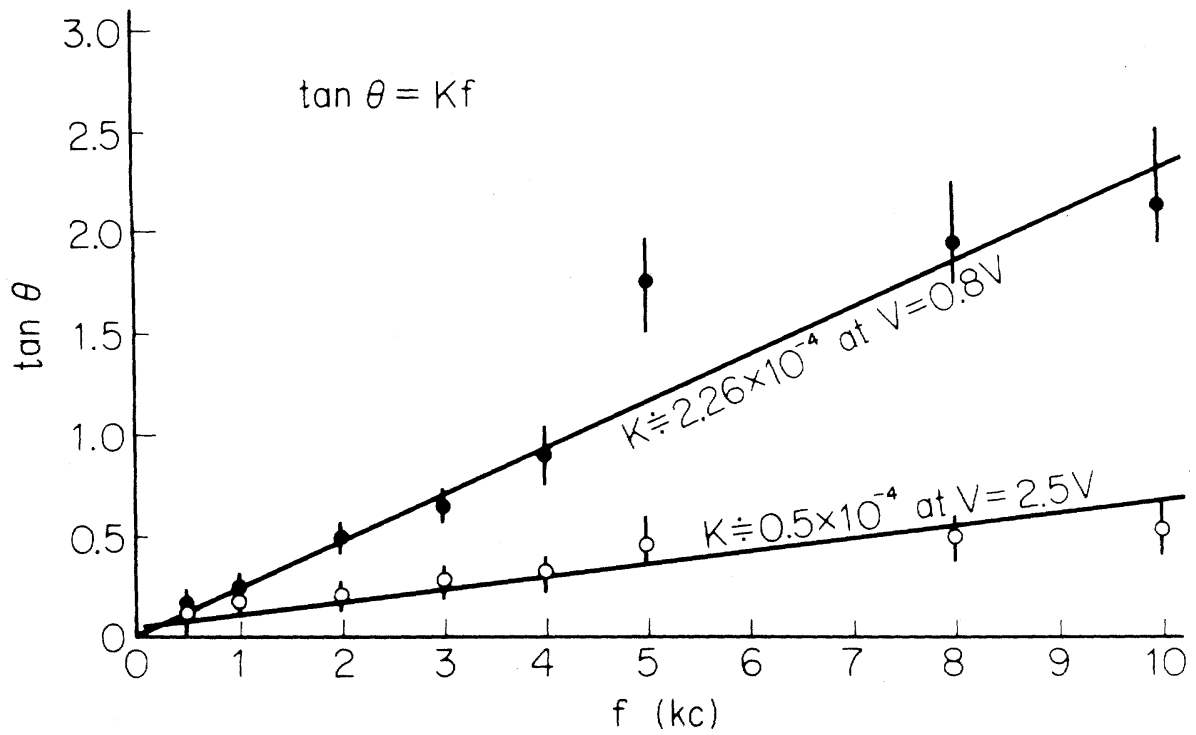

Fig. 10 Relation between the phase difference and the applied voltage 


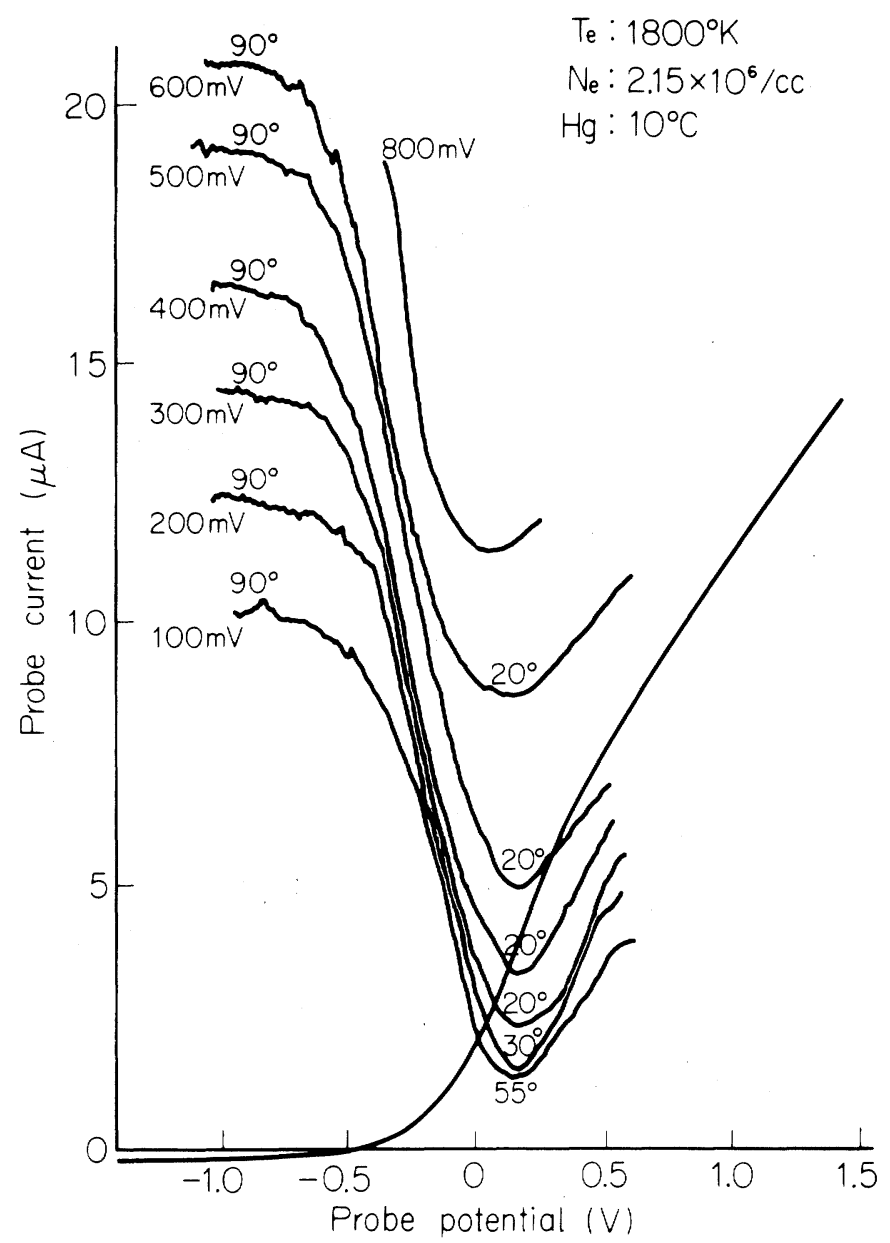

Fig. 11 Amplitude dependence of phase difference

\section{Theoretical Consideration of the Plasma Potential}

As is expected from the result in the section 2, the plasma potential may be determined by the probe potential, at which the sheath capacitance around the probe becomes maximum. To do this experimental determination, the ac signal of rather high frequency is used. The high frequency means to be higher than the ion plasma frequency in the test plasma. Grard (1965) proposed some kinds of models of the ion sheath around a probe. As one of these models, he proposed an ion sheath in which the ion distribution is homogeneous and the electron distribution is exponentially decreasing with the distance from the edge of the sheath. This type of ion sheath may correspond to the case in which the ion in the sheath is not rearranged by the high frequency voltage that is superposed to the probe. It can be said that this case corresponds to the second harmonic experiment using the high frequency described in the section 3. The electric potential in the ion sheath can be expressed by the following equation, 


$$
\nabla^{2} \phi=-\frac{n e}{\varepsilon_{0}}\left[1-\exp \left(\frac{e \phi}{k T_{e}}\right)\right]
$$

where $n$ is the electron density of the undisturbed ambient plasma, $e$ is the electronic charge, $\varepsilon_{0}$ is the dielectric constant of the vacuum, $k$ is the Boltzmann's constant, $T_{e}$ is the electron temperature, and $\phi$ is the electric potential.

Introducing new quantities $y=e \phi / k T, x=\frac{r}{\lambda_{D}}$ and $\lambda_{D}=\sqrt{\frac{\varepsilon_{0} k T_{e}}{n e^{2}}}$, eq. (14) can be expressed as follows,

$$
\frac{d^{2} y}{d x^{2}}=e^{y}-1
$$

Then the capacitance of the ion sheath around the probe, $C_{S}$, is determined as follows,

$$
C_{s}=\frac{A \varepsilon_{0}}{\lambda_{D}} \cdot \frac{e^{y}-1}{\sqrt{2\left(e^{y}-y-1\right)}}, \quad(y \leqq 0)
$$

The relation between $C_{S}$ and $y$ is shown in Fig. 12, It is expected from this figure that the capacitance becomes maximum at the probe potential which is equal to the plasma potential of the ambient plasma. Therefore, the second derivative method by using rather high frequency will give the space potential.

In the case of other models, in which the ion in the sheath can move corresponding to the superposed ac signal, the probe potential corresponding to the maximum sheath capacitance does not always fit to the plasma potential of ambient plasma.

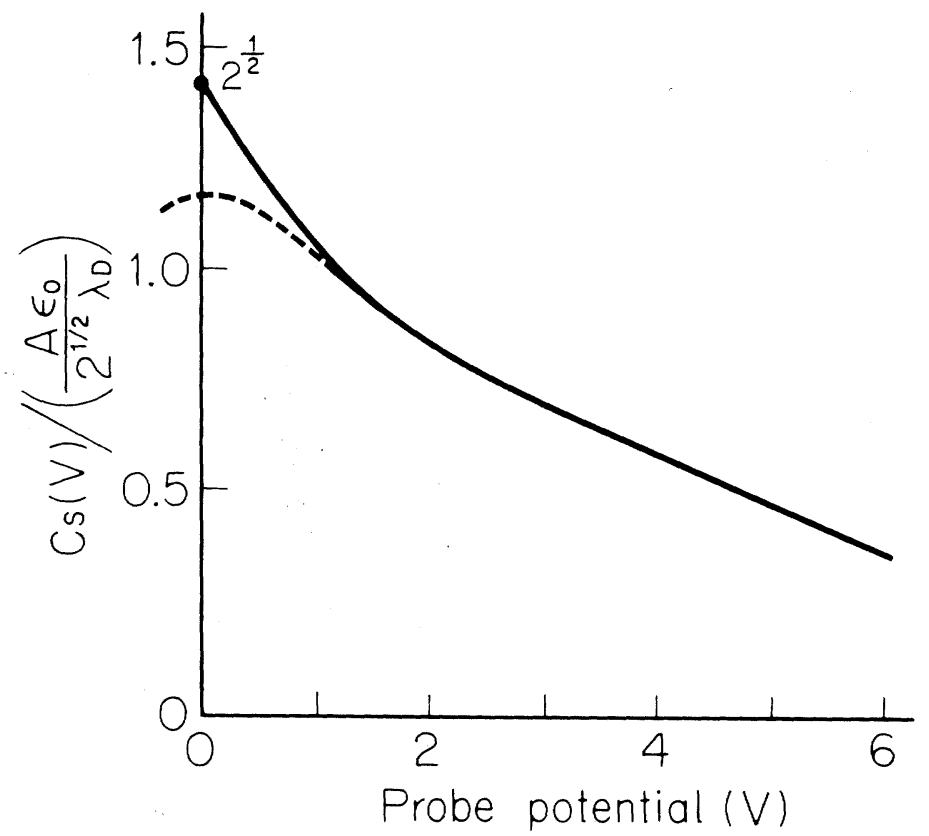

Fig. 12 Capacitance of the ion sheath. It is assumed that ions cannot follow the variation of alternating potential, and electrons decrease exponentially with respect to the probe potential. Probe potential is shown by the difference between probe and ambient plasma potential. 


\section{Conclusion}

In the present paper, the method of determination of plasma potential, especially by means of the second harmonic method is precisely discussed with the theoretical and experimental bases. This problem has not yet been discussed with enough theoretical consideration. It is concluded that the frequency of the ac signal which is superposed on the DC probe potential to detect the plasma potential, should be higher than the ion plasma frequency of the ambient plasma. In the determination of the electron energy distribution, the frequency lower than the ion plasma frequency of the plasma should be used.

The electron density which is determined from the electron saturation current at the plasma potential is compared with that is obtained by mean of the impedance probe. The saturation current is determined from the extrapolated probe current of the linear part of semi-logarithmically plotted curve of the Langmuir probe current at the plasma potential. The comparison is quite satisfactory, suggesting a high accuracy of the plasma potential determination by the second harmonic method.

\section{Acknowledgement}

Authors express their sincere thanks to Prof. T. Itoh for his instructive discussion and to Mr. H. Yamada for his continuous help to their laborious experiments.

\section{References}

Boyd, R.L.F. and N.D. Twiddy, Electron energy distribution in plasma, I. Proc. Roy Soc., A. 250, 53-69, 1958.

Dote, T., T. Ichimiya and M. Takeuchi, A new method for determination of the plasma potential by a "Twin Probe", J. Phys. Soc. Japan, 21, 2426, 1966.

Dote, T., A method for determination of the plasma potential by an RF Probe, J. Phys. Soc. Japan, 24, 224, 1968.

Druyvesteyn, M.N., Der Niedervoltbogen, Z. Phys., 64, 781-797, 1930.

Grard, R.J.L., Interpretation of impedance probe measurements in the ionosphere, Technical Report No. 2, Radio Science Laboratory, Stanford University, 1965.

Kemp, R.F. and J.M. Selen, Jr., Plasma potential measurements by electron emissive probes, R.S.I., 37, 455-461, 1966.

Kilvington, A.I., R.P. Jones and J.D. Swift, Effect of ac amplitude on the measurement of electron energy distribution function, J. Sci., Instr., 44, 5171967.

Medicus, G., Limitation for measuring the low-energy range of the electron spectrum by probe, A Survey of phenomena in ionized gases, Int. Atm. Energy Agency, 523-544, 1968.

Sato, M. and Y. Hatta, Glowing probe for space potential measurement in gas discharge, J.S.I. 39, 481482, 1962.

Takayama, K., New discharge tube and spherical mesh probe, Aeronomy Report, University of Illinois, No. 1, 26-29, 1963.

Verobéva, N.A., Yu. M. Kagan and V. M. Milenin, Electron velocity distribution in the positive column of a mercury discharge I, Soviet Phys., Technical Phys., 8, 1962. 